\title{
Germanica
}

\section{Un fugitif revient sur ses pas - Extraits}

\section{Aksel Sandemose}

Traducteur : Georges Ueberschlag

\section{CpenEdition \\ Journals}

Édition électronique

URL : http://journals.openedition.org/germanica/2935

DOI : 10.4000/germanica.2935

ISSN : 2107-0784

Éditeur

Université de Lille

Édition imprimée

Date de publication : 30 juin 1987

Pagination : 209-217

ISSN : 0984-2632

\section{Référence électronique}

Aksel Sandemose, "Un fugitif revient sur ses pas - Extraits », Germanica [En ligne], 1 | 1987, mis en ligne le 26 octobre 2015, consulté le 06 octobre 2020. URL : http://journals.openedition.org/ germanica/2935; DOI : https://doi.org/10.4000/germanica.2935

Ce document a été généré automatiquement le 6 octobre 2020.

(c) Tous droits réservés 


\title{
Un fugitif revient sur ses pas - Extraits
}

\author{
Aksel Sandemose
}

Traduction : Georges Ueberschlag

\section{NOTE DE L'ÉDITEUR}

Extraits traduits de En flykting krysser sitt spor, Aschehoug, 1955.

1 Maintenant je vais tout raconter. Et il me faut commencer par la fin, sinon je n'oserai jamais m'aventurer jusque là.

2 Un jour, j'ai assassiné un homme. Il s'appelait John Wakefield, et je l'ai tué, une nuit, il y a dix sept ans, à Misery Harbor.

3 À cette époque il y en avait pas mal qui sont devenus des assassins. La guerre mondiale faisait rage, mais il s'agissait de meurtres légalisés. Pour les victimes cela ne faisait guère de différence, mais pour le meurtrier il n'en allait pas de même...

4 Je viens de Jante, et là on m'a donné une conscience. Le plus horrible, c'était la nuit, lorsqu'il était debout dans la chambre. J'étais trop jeune pour pouvoir m'en débarrasser comme d'un événement malheureux auquel il n'y avait plus rien à changer...

5 Cela s'est passé à l'époque où l'on dit que la jeunesse bouillonne dans les veines, où le jeune homme chante comme un coq, mais où il lui arrive aussi très souvent d'être apathique et de se laisser aller à l'indifférence, tant il a été déçu. La jeunesse est ardente, et l'âge mûr est ardent pour ceux que la jeunesse n'a pas rendus stériles. La jeunesse, c'est l'âge où l'on apprend à connaître quel masque il faut porter. 


\section{Le péché, le pécheur et le bouc émissaire}

7 Lorsqu'un garçon avait commis une faute et qu'il n'était pas encore mûr pour la maison de redressement, on lui administrait le fouet à la salle de police. En présence du père et de quelques fonctionnaires qui servaient de témoins. Dieu sait si on a jamais réussi à faire un homme d'un tel garçon. Il y a des poètes qui ont essayé de créer des figures démoniaques. Mais aucun poète ne peut créer un démon qui soit pire que l'homme inconscient. Très vite je me suis rendu compte que le satan parfait devait être bête...

"Pour la peur et pour l'exemple", disait-on. Et la peur, nous la ressentîmes, mais l'exemple fut vain. On vous torturait, et tous les autres s'en trouvèrent malades de frayeur...

Christian Flaskhals, il a croupi presque toute sa vie dans la maison d'arrêt... Mon père hocha la tête d'un air grave en disant : Eh oui, déjà lorsqu'il était enfant, Christian s'est retrouvé à la salle de police. De la mauvaise graine.

10 C'est là une note bien salée que l'État doit payer pour qu'une demi-douzaine de sadiques puissent s'offrir parfois une distraction légale. Jante connait bien l'art de créer des boucs émissaires... J'ai une photo de notre classe à l'école. Souvent je la prends et je regarde les visages : celui-là, et celui-là... le sort aurait pu nous désigner tous.

\section{La grange d'Adamsen}

11 Il ne faut pas que tu franchisses la porte de chez Adamsen! C'était une phrase terrible. J'y ai beaucoup réfléchi, mais cela m'est resté un grand mystère. Souvent je crois que ce n'est pas tellement l'interdiction que je craignais, mais plutôt quelque chose de noir et d'oublié qui, un jour, s'est passé dans la grange d'Adamsen...

12 Quelque part au fond de moi-même je sens que je touche là un des grands axes de la vie... mais chaque fois que je crois l'avoir enfin atteint, le voilà qui a disparu. À vrai dire, je suis venu échouer ici sur les rives de l'inexplicable...

13 Oui, voilà ce qu'il en est, et cela ne te dit pas grand chose. À moi non plus d'ailleurs, je ne ressens qu'une grande et sauvage excitation, comme si toute la grange était en feu, comme si Jante tout entière était en feu, et que les murs tombaient, dans un nuage de poussière et de flammes. La destruction de Jante! Et pourtant quelque chose de tout autre, le contraire même. C'est le livre de l'Apocalypse...

Dans le mur qui entoure la ferme d'Adamsen sont scellées les tablettes sacrées... J'ai vu un jour une inscription - vu? je ne trouve pas d'autre mot: de sombres flammes qui formaient un demi cercle au-dessus des tablettes sacrées, et une fumée qui s'échappait de l'inscription : Tu ne dois pas entrer dans la maison de ton père.

Je m'arrête et je regarde l'obscurité de la grange. C'est en rampant comme des serpents que nous y sommes entrés... dans la grange de mon père. Oui, je marche là au bord de quelque chose que je ne connais pas. Mon père n'avait pas de grange du tout.

16 Je suis devenu un autre. Le fugitif a fait demi tour et veut revenir sur ses pas, mais il ne peut pas traverser le brasier de la grange d'Adamsen. Les années ont passé. Jante, c'est déjà loin, dans le temps et dans l'espace. Mais la grange est toujours aussi proche, et le feu y fait rage... cette grange d'Adamsen où, enfant, j'ai adoré Astarté, je m'y traîne en rasant le sol comme un rat. 
Dans la grange d'Adamsen, Agnès et moi nous avions aménagé une cachette dans le foin. Et nous avions mis des pommes dans le foin. C'était bon de goûter au fruit défendu dans la maison interdite. Il y avait une toute petite lumière dans notre trou, nous avions fabriqué une petite grille qui nous permettait de voir ce qui se passait en bas. Tout autour de nous il y avait des souris en pagaille. Et sur les poutres se promenait la belette, comme un étrange génie domestique.

C'était le grand bonheur.

... /...

21 J'entrevois autour du foyer de mon enfance les éléments de base d'une religion qui prend ses racines dans la terreur. La grange d'Adamsen les a tous réunis. Je vois des flammes, joyeuses et sinistres, qui montent, car le ciel et l'enfer se confondent dans la grange d'Adamsen... J'ai raté quelque chose d'important. Si je pouvais traverser le brasier de la grange d'Adamsen, j'y trouverais la racine la plus profonde de ce qui s'est passé à Misery Harbor. Maintenant je peux te le dire, il en est vraiment ainsi. Je ne peux pas le prouver, mais je l'affirme pourtant, car quelque chose au fond de moi me dit que c'est la vérité. Si je pouvais y pénétrer, j'en ressortirais omniscient...

Je ne traverse pas le feu. Néanmoins, tout ce que je t'ai raconté, et tout ce que je te raconterai encore fera que la grange d'Adamsen sera démolie jusque dans ses fondements, car j'ai une clé pour ouvrir même le dernier réduit. Un jour, j'étais si près du feu qu'il m'a roussi les cheveux. C'était lorsque j'ai vu John Wakefield pour la dernière fois, la fois où il m'a tourmenté et poussé tellement à bout que tous les démons se sont déchaînés. Il y avait un arc de feu tout autour de nous lorsque cela s'est passé, c'était la grange d'Adamsen qui brûlait à Misery Harbor, et des lettres de feu surgissaient de l'arc de lumière qui consumait tout :

$\mathrm{Tu}$ vois, je connais une part de ton secret !

\section{Commentaires de la loi de Jante}

23 C'était la différence que l'on ne supportait jamais. Et cela créait une tension insoutenable. Tous ils criaient comme des fous à l'oppression, et tous ils opprimaient à leur tour, comme un simple maillon de la longue chaîne répressive que les siècles ont reçue en héritage.

On peut s'imaginer qu'un jour, il y a bien longtemps, les habitants de Jante ont compris quelles étaient leurs capacités. Ils n'ont pas dû les estimer très grandes, ou alors il leur était impossible d'en concevoir d'autres. Ils en firent donc les frontières de Jante, et celui qui voulait passer outre était un traitre à Jante. Et sur ces capacités immuables ils établirent leur morale. Celle-ci prit force de loi, et la loi dégénéra en religion.

La loi de Jante et la religion de Jante nous montrent que des hommes qui ont été suffisamment longtemps sous le joug deviennent à leur tour leurs propres tyrans. Jante s'est donné à elle-même sa propre loi et courbe le dos, sans que cela ne doive créer des problèmes pour qui que ce soit...

Celui qui a vécu sous la loi de Jante durant les quinze ans où il aurait dû essayer de devenir un homme - c'est-à-dire d'aimer son prochain - en ressent le poids sanglant et la volonté hystérique de puissance... 
27 Avec la loi de Jante les hommes tuent toutes leurs chances... Elle les frappe tous, ils se tordent sous son joug et ils l'appliquent de façon impitoyable... Chacun est suspendu à sa croix, et il n'a pas besoin d'un autre symbole. Tous ils hurlent sur leur croix, suant sang et eau, tous ils se tordent de douleur et lancent d'une voix venimeuse à leur frère crucifié : Crois-tu peut-être que quelqu'un va se soucier de toi? La loi de Jante n'était pas simplement la loi, elle était au cœur de la langue elle-même, tout ce qui était dit pouvait se ramener à elle... On pouvait mettre fin à n'importe quelle discussion avec un paragraphe quelconque de la loi de Jante. Parfois on était assis autour d'une tasse de café, et il suffisait d'un simple regard, je m'en souviens, pour que les paroles de la loi nous couvrent tous de leur étreinte suffocante, et deviennent l'air même que nous respirions, un air qui ne se nourrissait que de rêves et de vengeance...

Il n'y avait que peu de choses dont on pouvait parler à Jante, et seuls les ainés pouvaient aborder ces sujets dans les assemblées, en parlant lentement, d'une voix mal assurée, tandis que les plus jeunes restaient assis, les yeux baissés, entassant leur haine. S'il leur arrivait de poser une question, il fallait l'accompagner de mille précautions: Nous, évidemment, nous ne savons rien, mais toi qui es si sage - et la réponse nous parvenait alors de l'olympe. Car celui-ci connaissait toujours le pourquoi. Le contraire eut été impensable. Mais même si le petit bonhomme n'avait pas droit à la parole, il arrivait bien des fois qu'il se marre au fond de lui-même en écoutant les réponses qu'on lui faisait.

29 Comment un être peut-il tremper son âme dans un tel milieu, comment Jante peut-il produire autre chose que des esclaves et de temps à autre un scorpion ou un assassin ? Le Bon Dieu là-haut, que peut-il bien faire d'une telle engeance à l'humeur éternellement visqueuse, prête à bondir en voyant quelqu'un, n'importe qui, en train de se dorer sur un nuage en s'imaginant qu'il est quelque chose.

30 L'union fait la force. Jante réussit à maintenir Jante sous le joug. Ce que nous avons pu souffrir, et comme nous nous sommes tordus de douleur ! Tous sont également grands, mais tous croient que les autres sont plus grands. Ce sont les assises de la cité. Il est devenu impossible pour un individu de s'insurger il met bien trop longtemps à décrouvrir qu'il a été dompté, si jamais il le découvre un jour. La plupart ne le font jamais. Et ce sont justement eux, les infatigables trimeurs des usines, ces ouvriers standardisés, utiles et taillés sur mesure, qui n'ont fait qu'aller et venir trois fois par jour pendant trois générations, jusqu'à ce qu'ils n'en pouvaient plus. La fourmi et l'abeille se sont mieux établies que l'homme, de façon moins impitoyable...

31 Je dois le dire, je ne suis pas devenu un type bien épatant. Pas le genre innocent persécuté, loin de là. Peut-être que je m'en rends tellement compte que, par moments, je me noircis un peu plus que nécessaire. Je n'étais pas innocent. Mais l'innocence est quelque chose que l'on vous arrache, et qui est-ce qui me l'a arrachée ? Peu à peu toute cette vie n'était plus faite que de vengeance et de démesure, une tragédie que l'on rejouait sans cesse jusqu'à ce que, pour ma part, je mette les voiles.

32 Nous nous sommes fait beaucoup de mal. Mais le temps n'est plus à l'expiation et au pardon, là où tous ces malheurs sont arrivés, et cela ne servirait plus à personne. Là-bas cela constitue un recueil de contes achevé, fermé à tout jamais, même si quelques gouttes de sang tombent encore des feuilles...

33 Il est à peine croyable que des hommes puissent passer leur temps à des choses pareilles. Leur temps ? C'est plutôt le creuset barbare et impitoyable où se décide notre 
sort, si nous allons devenir des hommes ou des bêtes de somme. La plupart deviennent des bêtes de somme, et ils veulent que les autres le soient aussi. Tu crois peut-être que tu es plus que nous?

34 Je préfère les fourmis. Elles ont du style. Le dieu des fourmis, tu peux lui faire confiance.

\section{Le Hollandais Volant et Klabautermann}

Le cheval, lui, ne me faisait pas peur, il me remplissait d'une sainte admiration. Et voistu, j'ai retrouvé le cheval dans un autre personnage, celui du Hollandais volant.

C'est la légende du Hollandais qui me fit penser un jour à la première impression que me firent les chevaux dans mon enfance. Cette atmosphère qui entoure le Hollandais en pleine mer, je l'avais déjà connue durant les premières années de ma vie, à la vue des étalons de la ferme d'Adamsen.

On pense généralement que le Hollandais volant inspire la peur. Ce n'est pas vrai du tout. On l'admire jusqu'à l'extase. C'est l'homme-catastrophe devenu vision, le grand chambardeur. Soit, il ne gagne pas à provoquer Dieu lui-même, mais à le berner, si !...

Combien de gens songent-ils à ce fait qu'il veut constamment contourner un cap ? Le Cap Horn, le Cap de Bonne Espérance, et n'est-ce pas une étrange et immense farce qu'il n'y arrive jamais? Oui, pourquoi Adam n'a-t-il pas eu le droit de prendre une pomme? Le Bon Dieu a ses idées. Tu ne dois pas toucher à la pomme... jamais, absolument jamais tu ne dois contourner le Cap Horn... Évidemment, la Bible nous est venue d'Orient. Si elle avait été écrite sur les rives de l'Europe du Nord-Ouest, Mademoiselle Nibe (l'institutrice, N.d.T.) nous parlerait aujourd'hui de ce satané Hollandais qui nous a valu le péché originel parce qu'il a voulu braver la puissance du père et voguer tout autour de la Ville. Au lieu de cela on nous raconte l'histoire d'Ève et de la pomme, mais nous, nous aimons le Hollandais et sa révolte.

39 Cette sauvage atmosphère de catastrophe qui entoure le Hollandais, je la connaissais déjà dans la grange d'Adamsen, ce grand bonheur ardent! D'abord il y avait le cheval, et c'est assis sur son dos que je suis entré dans la grange d'Adamsen. Et plus tard, en voyageant à travers le monde, j'ai encore rencontré le cheval : ce fou qui cherchait à contourner le Cap Horn.

Sur la mer on rencontre aussi un autre homme, qui n'a ni chance ni bateau. C'est le noir Klabautermann. Lui, je l'ai bien reconnu, c'était l'effrayant spectre de la grange. Il est le maudit, sans cheval et sans bateau - et en face nous avons le Hollandais volant, le mécréant fumant qui a son bateau, sa bonne chance et qui doit contourner le Cap Horn. Ensemble ils sont la grange d'Adamsen.

41 Je me suis demandé très longtemps, qui il était vraiment, qui l'avait créé et pourquoi... et je suis tombé par hasard sur une vieille image naïve de Klabautermann, il était petit et s'appuyait contre un mât. Il portait un bonnet pointu rouge, une longue barbe, et dans la main il tenait un marteau. Ce marteau me rappela quelque chose, mais ce n'était pas si facile à combiner avec le personnage.

42 La source suivante, ce fut un marchand de chevaux allemand, un ancien marin. Nous étions assis dans un estaminet à Malmo, et il me raconta une sinistre histoire à propos de Klabautermann qui s'était installé, disait-il, dans le grand mât d'un navire en 
provenance de Konigsberg. Chaque nuit il sortait de son mât et fracassait le crâne d'un homme avec son marteau.

Le marteau, encore lui, et il avait commencé à servir ! Je fis une remarque à propos de ce marteau, et le marchand de chevaux me répondit: Ah oui, le Klabautermann, c'est un mot basallemand qui signifie l'homme au marteau...

Je songeais à ce qu'il était advenu de Tor, le dieu au marteau. Les prêtres l'avaient sans doute expédié en haute mer, avec d'autres figures païennes, et je comprenais à présent pourquoi il avait un bonnet pointu, la foi populaire l'avait peu à peu assimilé aux lutins et autres êtres nocturnes. J'en conclus que Klabautermann, c'était le dieu Tor déchu...

Avant que le christianisme ne vienne dans les pays nordiques, on n'adressait pas de prières aux dieux, mais on concluait une alliance avec eux. On était des contractants. Cela se perpétua assez longtemps, sous une forme bâtarde mais assez visible, dans les pactes avec le diable. Faust est un drame païen. Mais il arrivait que les dieux refusent de conclure un pacte. D'où l'apparition de deux types de personnages, le chanceux et le guignard... Ils sont toujours là, ils s'appellent le Hollandais volant et Klabautermann... Ils sont à la base de tout ce que Mademoiselle Nibe nous a enseigné, ils resurgissent sous de nouvelles formes dans chaque âme d'enfant. Puis ils se confondent, et on n'arrive plus jamais à les séparer... La grange d'Adamsen est une et indivisible, et Janus y est le maître. Il ne sait jamais d'avance avec quelle bouche il va parler.

Exiger qu'ils soient séparés, c'est ça l'utopie.

47 J'ai admiré John Wakefield. Il était tout ce que j'avais obstinément cherché à devenir... Il était le puissant, il était le chanceux...

Lorsque le malheur vint s'abattre sur nous, ce fut la rencontre du Hollandais volant et de Klaubautermann. Comme un éclair surgi de mon ciel d'orage qui vint brûler les sabots de l'étalon d'Adamsen. Ce fut une rencontre entre deux hommes telle que nous la souhaitons au plus profond de nous-mêmes. Je veux posséder toutes les femmes et tuer tous les hommes. Nous sommes des chasseurs de têtes.

\section{Halfway Mountain}

Il y a une montagne à l'intérieur de Terre-Neuve, qui s'appelle Halfway Mountain. Elle s'élève dans une région de forêts assez plate, et paraît donc plus haute qu'elle ne l'est en réalité. Je l'ai approchée lors d'une expédition de chasse, et j'ai remarqué combien une telle montagne pouvait changer complètement d'aspect chaque fois que l'on changeait de place, ne fût-ce que légèrement. On peut avoir des centaines de descriptions de Halfay Mountain, et toutes sont pareillement justes. Je ressens un besoin intense de te l'avouer maintenant, la montagne est grande, elle a des aspects variés, mais celui qui était couché par terre dans ses chaînes n'a vu Halfway Mountain que de l'endroit où il était couché. 